\title{
AIR IN THE EYE*
}

BY

\section{A. LISTER}

\section{London}

THE introduction of air, or saline, into the eye is a fairly recent innovation. The practice has its advocates and its critics, so that a short review of its uses by one of the former may be of interest. The author's experience, chiefly with air, forms the basis of this paper. Recently, however, it has become apparent to him that there are occasions when saline is preferable. In the conclusion, therefore, the relative merits of air and saline are briefly discussed.

In the anterior chamber, the most common use of air is probably after cataract extraction. It has even been stated that the injection of air is the most important part of the operation! In most instances it is unnecessary because, with the use of sutures-which again is a controversial matter-the anterior chamber reforms rapidly. There are, however, occasions when the iris is lacking in tone and when at the end of the operation it seems wise to push it away from the section with a bubble of air. This applies to both extra- and intra-capsular extractions.

The only likely serious complication is the passage of the air through the pupil, behind the iris, where of course it does the opposite of what is desired. The writer was first made aware of this possibility in a patient in whom a large bubble of air entered in the "wash-out" and filled the anterior chamber. All looked so satisfactory that it was decided to leave it there and the patient went back to bed in Fowler's position. Next day the iris was found to be hard up against the section with no sign of any air. It seemed advisable to . abscise the threatened prolapse and the patient came back to the theatre for this to be done. As she lay flat on the table a bubble of air emerged through her pupil into the anterior chamber. Even then what had really happened was not understood and the abscission was completed. It was only later, when a rather similar event occurred in another patient, that the problem was solved.

This danger is sufficient to antagonize some surgeons against the use of air after a cataract operation, but it is quite easily avoided by ensuring that the patient is kept reasonably flat-with not more than three pillows instead of a bed-rest for the first day or two after operation.

Air is probably contraindicated where there has been a complete

* Received for publication, November 9, 1956 
iridectomy, and certainly where there is vitreous loss. On one occasion when there was vitreous loss after an attempted linear extraction of a congenital cataract the only effect of the air was to push more vitreous out.

Air can be used only if the section is closed with sutures and there are many who consider sutures and, still more, air to be unnecessary complications of the cataract operation. The answer, as far as the writer is concerned, is that they have made iris prolapse an extreme rarity and have allowed us to take liberties with elderly patients-such as leaving the pupil intact and getting them up the day after operation-which would have been a serious risk in pre-suture days.

Another operation in which air is useful on occasions is penetrating keratoplasty. As is well-known there is seldom a risk of an anterior synechia provided that during the operation the pupil border is either inside or outside the edge of the graft, so that for grafts of $5 \mathrm{~mm}$. one likes to operate with a large pupil, and for grafts of $6 \mathrm{~mm}$. or more one operates with the pupil contracted by miotics. There are, however, some pupils which, in spite of intensive pre-operative miotics, dilate under the influence of pantocaine and the retrobulbar anaesthetic, usually to about the same size as the graft. In such cases, and in those in which the pillars of an iridectomy cross the edge of the graft, it is an advantage to make sure that the iris is clear of the graft at the end of the operation with a few bubbles of air. If one uses direct sutures there is no risk of the graft being displaced by the air. The air may be introduced with a No. 20 needle with the point filed off, inserted along the track of a small paracentesis puncture made early in the operation-usually just after marking the recipient cornea with the trephine.

After total keratoplasty, too, one may follow Castroviejo's instructions and fill the anterior chamber with air, so as to keep the vitreous away from the graft.

Stallard (1955) recommends that, in graft operations where the anterior chamber is shallow or has been lost through a too deeply inserted "preplaced" suture, it may be deepened or restored so as to make trephining the cornea safe by means of air injected through a cyclodialysis incision.

A third operation which may be completed with advantage with the injection of air into the anterior chamber is cyclodialysis, as advocated by Rome and Koff (1948). The air, besides hastening the re-formation of the anterior chamber and thus, one hopes, keeping the cyclodialysis cleft open, stops haemorrhage if there is any. It acts, as the authors quoted put it, like an "internal tourniquet". The air is introduced through the cyclodialysis opening with a fine lacrimal cannula. After goniotomy too, which is similar in a way to cyclodialysis, reformation of the anterior chamber immediately after the operation does at least theoretically help to prevent the re-formation of goniosynechiae. Incidentally, it is sometimes possible to reform the anterior chamber with air after goniotomy by merely "squirting" the air at the site of entry of the goniotomy needle. 
Some years ago it was suggested that goniotomy could be facilitated by replacing the aqueous with air, thus giving a view of the angle without having to use a gonioscopic lens. Unfortunately the contact of the bubble with the posterior surface of the cornea makes a practically opaque screen through which details of the angle are invisible.

Another occasion in which air is of great value-perhaps its greatest-is in the repair of a perforated eye, when it is often extremely difficult to ensure that uveal tissue, lens capsule, or vitreous is clear of the wound. This point is best illustrated by a case report.

A small boy was cutting a piece of string with a nail file when the file slipped and perforated the eye, making a jagged wound involving both cornea and sclera. The lens was badly damaged and there was prolapse of lens and iris from the wound. At operation the prolapse was abscised as effectively as possible and the lens capsule was drawn out of the wound followed by a "bead" of vitreous. The wound was then closed with direct sutures.

Clearly it wasequite impossible to free the wound entirely of intra-ocular tissue, and one was faced with the prospect of extensive anterior synechiae and an exceedingly dangerous eye. Luckily it was possible to restore most of the anterior chamber by injecting air through the wound. Ten days later there was still a small band of capsular synechia. This was divided, but in doing so the wound opened slightly and the anterior chamber was lost. However, it was this time completely reformed by an injection of air through a paracentesis puncture. The eye settled down well and though it has grossly defective sight is at least a cosmetic asset. It would certainly have been lost without the use of air.

This case illustrates the important place that air has in the treatment of penetrating wounds and, incidentally, of anterior synechiae. In the latter instance, the chances of recurrence are greatly lessened if, after the synechia has been divided, the anterior chamber is re-formed with air injected through a small "pre-placed" paracentesis puncture.

One might add that the use of air has largely been made possible by the exceedingly fine suture materials-needles and silk-which are available to us nowadays. These have, in the writer's view, rung the knell of the conjunctival flap-never a satisfactory method of closing a penetrating wound.

Lastly, as far as the anterior chamber is concerned, is the use of air in restoring the chamber when its re-formation is delayed after a filtering operation or after cataract extraction. The writer has been fortunate in having little experience of this complication in the latter, but Kronfeld (1954) speaks of it as being fairly common and advocates the injection of air into the anterior chamber combined with drainage of the choroidal detachment which is a frequent complication.

The writer's experience with filtering operations has been less happy, and to him delay or failure of re-formation of the anterior chamber after a trephine has, in elderly patients, been a discouragingly common complication. The answer probably lies in prevention by modification of technique. However that may be, in the few cases in which restoration of the anterior chamber with air has been attempted it has never lasted more than a day or two. 
In the posterior chamber the use of air in the treatment of retinal detachment is becoming increasingly common. It may be illustrated by the following three cases:

An elderly stout woman with an upper quadrant detachment would have found the normal posture, on her back, quite impossible. She was therefore nursed in Fowler's position, and the large bubble of air which was injected into the vitreous at the end of the diathermy operation kept her retina in place and it became firmly re-attached.

An elderly man, who also had a detachment with an upper quadrant tear, found it quite impossible to keep still in bed lying on his side, and a week after operation, although the retinal hole appeared to be covered by diathermy, there was a large temporal balloon which seemed to be increasing. Without further diathermy, the balloon was tapped and air injected into the vitreous, after which the patient was nursed in Fowler's position, with a most satisfactory result.

A boy aged 12 had a total detachment in which no hole could be found. A full circle lamellar scleral resection was performed in two stages, air being injected at the end of each operation. After each the retina was entirely replaced for a short period but subsequently redetached. A third attempt, combining a diathermy barrage with air injection, again replaced the retina, this time permanently. This case shows that by means of air a total detachment may be so far replaced as to give it some chance of adhesion where adhesion is necessary, thus improving the prognosis in a type of case which has generally been considered hopeless.

These three cases illustrate two obvious ways in which air may be of assistance, namely in overcoming gravity in the elderly patient and in "controlling" a totally detached retina. It has a third possible action which is worth mentioning; that is of breaking down adhesions between vitreous and retina which would prevent the replacement of the latter.

The effect of an air injection upon the vitreous itself is not known. Presumably it is not beneficial, although in most cases the vitreous is already degenerate. There is no question therefore of advocating air injection in more than a minority of cases of retinal detachment, and it is, in fact, contraindicated when there is a particularly large tear owing to the risk of the air passing behind the retina. In aphakia there is a risk of the air coming forward into the anterior chamber. This might not matter but it can be prevented by suitable positioning of the patient.

Redmond Smith (1952) reported success in three out of five difficult cases in which air was injected into the suprachoroidal space instead of into the vitreous.

\section{Technique}

The technique of air injection is simple. Sterility may be achieved either by autoclaving already loaded syringes or by drawing up the air through a flame or through sterile cotton wool.

A No. 20 needle with the point filed off is satisfactory for injecting air into the anterior chamber through the wound of either operation or injury or through a pre-placed paracentesis puncture. In cyclodialysis a lacrimal needle is preferable because its smooth tip is less likely to penetrate or injure the ciliary body. In 
retinal detachment the injection is made into the vitreous, after the subretinal fluid has been tapped, with a sharp No. 20 needle inserted $8 \mathrm{~mm}$. from the limbus -i.e. through the region of the pars plana of the ciliary body. It facilitates the entry of the needle to make a small preliminary incision half or three-quarter way through the sclera, and the eye may be fixed by grasping one edge of this incision with fine-toothed forceps. Otherwise piercing a soft eye is apt to be difficult. Another aid is to have a small "stop" of solder on the shaft of the needle about $3 \mathrm{~mm}$. from its point. This prevents the needle going in too far.

Choice between Air and Saline.-In retinal detachments, particularly those in the elderly when the tear is in the upper part of the eye, air seems to be the obvious choice, though this is not the view of everyone. In the anterior chamber, air has the advantage that owing to its greater surface tension it is less likely to leak than saline. This surface tension may, however, sometimes be a disadvantage, preventing the air from spreading where one wants it. For example, in a corneal graft, a bubble of air, in spite of efforts to dislodge it, remained localized in one part of the anterior chamber, forcing the iris elsewhere against the back of the graft. It was therefore withdrawn and saline introduced in its place, with good results. A further point is, that being more foreign to the eye than normal saline, air may be more irritating.

Opinions differ among different people and vary from time to time in the same person. That of the writer at the present time is that saline is to be. preferred when it fulfils the desired purpose.

KRONFELD, P. C. (1954). Amer. J. Ophthal, 38, 453 .

\section{REFERENCES}

Rome, S., and Koff, R. (1948). Arch. Ophthal. (Chicago), 40, 134.

SMITH, REDMOND (1952). British Journal of Ophthalmology, 36, 385.

Stallard, H. B. (1955). Trans. ophthal Soc. U.K., 75, 33.

\section{Postscript}

Since this article was written the author has encountered a complication of which he had heard but had not experienced, namely air-lock of the pupil. It occurred in a case in which air had been introduced after a cyclodialysis. On the following day the pupil was found to be sealed by the air bubble with consequent iris bombé and raised tension. The situation was relieved by paracentesis.

This accident was obviously possible in all cases where the iris is intact and provides a further point in favour of saline. It is probable, however, that it may be avoided by limiting the amount of air introduced and by positioning the patient so that the air does not completely cover the pupil. 